\title{
pH-Regulated Synthesis of Trypsin-Templated Copper Nanoclusters with Blue and Yellow Fluorescent Emission
}

\author{
Jie Feng, ${ }^{\dagger}$ Yonglei Chen, ${ }^{\dagger}{ }^{\dagger}$ Yangxia Han, ${ }^{\dagger}$ Juanjuan Liu, ${ }^{\dagger}$ Sudai Ma, ${ }^{\dagger}$ Huige Zhang, ${ }^{\dagger}$ \\ and Xingguo Chen $*,+\neq 0$
}

${ }^{\dagger}$ State Key Laboratory of Applied Organic Chemistry and Department of Chemistry and ${ }^{\ddagger}$ Key Laboratory of Nonferrous Metal Chemistry and Resources Utilization of Gansu Province, Lanzhou University, Lanzhou 730000, China

Supporting Information

ABSTRACT: In this article, a simple protocol to prepare water-soluble fluorescent copper nanoclusters (CuNCs) using trypsin as a stabilizer and hydrazine hydrate as a reducing agent was reported. It was found that the $\mathrm{pH}$ of the reaction solution was critical in determining the fluorescence of CuNCs. CuNCs with blue and yellow fluorescent emission were obtained under basic and acidic conditions, respectively. Although the detailed formation mechanisms of these CuNCs required further analysis, the synthetic route was promising for preparing different fluorescent metal NCs for applications. With good water solubility and excellent photostability, the yellow-emitting CuNCs could serve as a fluorescence probe for detection of $\mathrm{Hg}^{2+}$ based on the aggregation-induced quenching mechanism. The fluorescence quenching efficiency had fantastic linearity to $\mathrm{Hg}^{2+}$ concentrations in the range of $0.1-100 \mu \mathrm{M}$, with a limit of detection of $30 \mathrm{nM}$. Additionally, the yellow-emitting CuNCs exhibited negligible cytotoxicity and were successfully applied to bioimaging of HeLa cells.

\section{INTRODUCTION}

Metal nanoclusters (MNCs), consisted of several to hundreds of metal atoms, have drawn considerable attention due to their unique physical, chemical, and optical properties resulting from their discrete energy levels and band-gap energy structures. ${ }^{1,2}$ In particular, compared with conventional organic fluorophores and semiconductor quantum dots, fluorescent MNCs exhibit several advantages such as strong photoluminescence, good biocompatibility, excellent photostability, and sub-nanometer size. ${ }^{3}$ Thus, they have been developed to be used in a wide range of applications in sensing ${ }^{4,5}$ and imaging. ${ }^{6,7}$

Among the studied MNCs, gold NCs (AuNCs) and silver NCs (AgNCs) have received extensive research attention by size-controlled synthesis, structural characterization, and property investigations. In fact, compared with gold and silver, copper was more popular in industry because of its high conductivity and much lower cost. Nevertheless, over the past decades, studies on the synthesis, properties, and applications of copper NCs (CuNCs) were scarce primarily because of their susceptibility to oxidation and the difficulty in preparing extremely tiny particles. ${ }^{8}$ In recent years, considerable efforts have been devoted to exploring the synthesis of fluorescent $\mathrm{CuNCs}$ and great progress has been achieved. By employing a series of scaffolds or capping agents, such as small molecules, ${ }^{9}$ polymers, ${ }^{10}$ oligonucleotides, ${ }^{11}$ peptides, ${ }^{12}$ and proteins, ${ }^{13}$ stable CuNCs have been successfully prepared. Among these methods, protein-templated synthesis is particularly attractive as proteins could serve as environmentally benign reducing and stabilizing molecules. However, there were few reports on the discussion of the mechanism for the formation of CuNCs and it remained unclear how the protein template affected the CuNC fluorescence behaviors under various reaction conditions. In a previous report, the $\mathrm{pH}$-dependent synthesis of pepsin-AuNCs with different fluorescent emission was developed. The different charges on pepsin under different $\mathrm{pH}$ conditions affected the structure of pepsin chains, which led to the formation of AuNCs with different fluorescent emission. ${ }^{14}$ Therefore, it enlightened us whether multicolored CuNCs could be prepared by regulating the reaction $\mathrm{pH}$.

$\mathrm{Hg}^{2+}$ is one of the most toxic heavy-metal ion pollutants that exists in water, soil, and food. Mercury can accumulate in organisms and has long-term adverse effects on liver, kidney, central nervous system, and so on. Therefore, developing effective methods for the sensitive and selective detection of $\mathrm{Hg}^{2+}$ was especially important for environmental monitoring and clinical research. Traditional methods of $\mathrm{Hg}^{2+}$ sensing, including atomic absorption/emission spectroscopy, inductively coupled plasma mass spectrometry, stripping voltammetry, etc. $^{15-18}$ were limited by the disadvantages of requiring expensive instruments, the complex procedures in sample preparation, a specific worker, etc. Electrochemical, colorimetric, and fluorescent sensors for $\mathrm{Hg}^{2+}$ have also been

Received: July 24, 2017

Accepted: November 30, 2017

Published: December 19, 2017 


\section{Scheme 1. Illustration of the Synthesis of CuNCs with Blue and Yellow Emission}
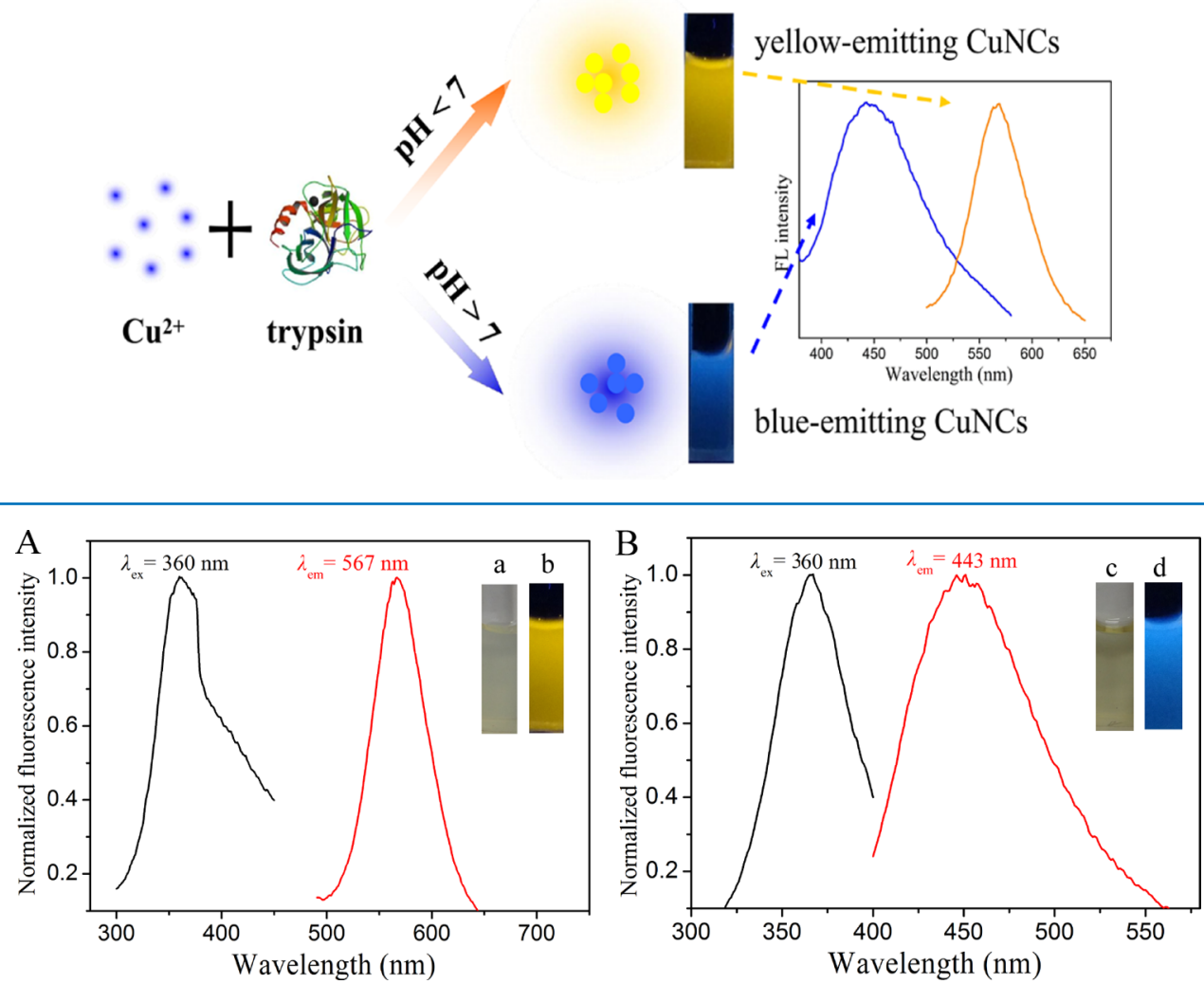

Figure 1. Fluorescence excitation and emission spectra of the yellow- (A) and blue- (B) emitting CuNCs; insets show photographs of the CuNC solution under visible $(\mathrm{a}, \mathrm{c})$ and $\mathrm{UV}(\mathrm{b}, \mathrm{d})$ light irradiation.
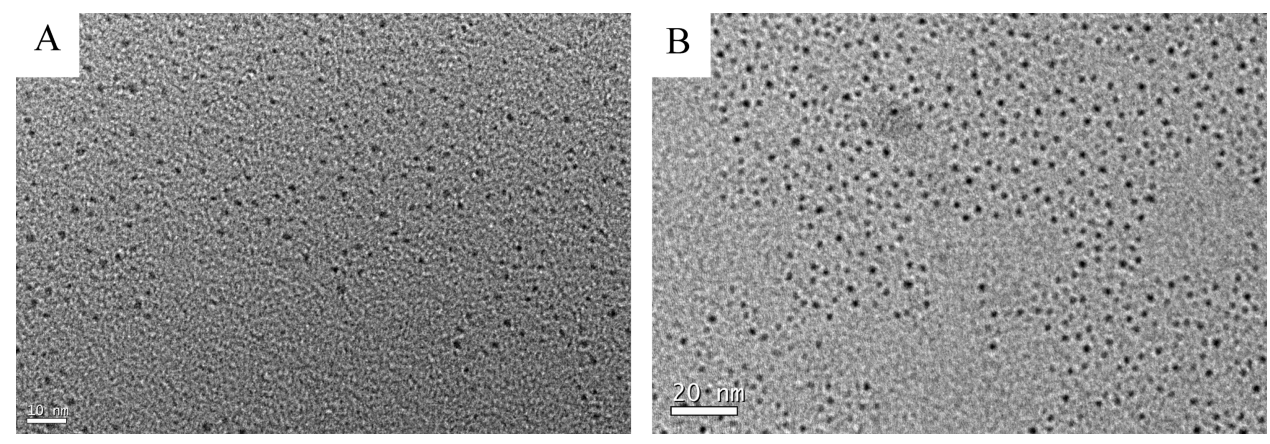

Figure 2. TEM images of the blue-emitting (A) and yellow-emitting (B) CuNCs.

reported over the past decade. ${ }^{19-29}$ Among these methods, fluorescent $\mathrm{Hg}^{2+}$ sensors based on various nanoparticles have been developed due to their unique advantages such as high sensitivity, simple operation, and fast response. ${ }^{30-33}$

On the basis of the above conditions, we reported for the first time a $\mathrm{pH}$-dependent synthesis of CuNCs with blue and yellow fluorescent emission using trypsin as a template and hydrazine hydrate as a reducing agent (Scheme 1). Trypsin is an important digestive enzyme produced by pancreatic acinar cells. ${ }^{34}$ It is also a good candidate for synthesis of trypsinstabilized CuNCs as trypsin is rich in amino acid residues, with 7 cysteine (Cys) and 10 tyrosine (Tyr) residues. The different conformational states of the trypsin molecule under different $\mathrm{pH}$ conditions could affect the interaction between trypsin and copper ion surface, leading to the formation of CuNCs with different sizes at different $\mathrm{pH}$ conditions. Then, the prepared yellow-emitting CuNCs were successfully employed as an effective fluorescent probe for $\mathrm{Hg}^{2+}$ sensing. Because of the low toxicity and good biocompatibility of the yellow-emitting CuNCs, they were also used in cell labeling of HeLa cells.

\section{RESULTS AND DISCUSSION}

Trypsin contained rich Cys, His, and Tyr that could act as chelating groups for sequestering copper ions and polyvalent ligands for passivating the surface of metallic materials. ${ }^{35} \mathrm{Next}$, the reducing agent $\mathrm{N}_{2} \mathrm{H}_{4}$ was applied to quickly reduce $\mathrm{Cu}^{2+}$ cations to CuNCs. It has been reported that proteins exhibit different conformational states at different $\mathrm{pH}$ levels, which could affect the size and fluorescence properties of MNCs. ${ }^{14}$ Therefore, it was of interest to investigate the synthesis of trypsin-templated $\mathrm{CuNCs}$ at different $\mathrm{pH}$ values. In a typical synthesis, trypsin and $\mathrm{CuSO}_{4}$ solution was mixed thoroughly. 
For the synthesis of yellow- and blue-emitting CuNCs, the $\mathrm{pH}$ of the mixture was adjusted to 3.4 and 12.0 , respectively, before addition of $\mathrm{N}_{2} \mathrm{H}_{4}$. It was worth noting that after addition of $\mathrm{N}_{2} \mathrm{H}_{4}$ to the mixture, the solution $\mathrm{pH}$ changed from 3.4 to 5.1 for the yellow-emitting CuNCs and remained unchanged for the blue-emitting CuNCs. After incubating at $70{ }^{\circ} \mathrm{C}$ for $2 \mathrm{~h}$, $\mathrm{CuNCs}$ with different fluorescent emission were obtained. Figure 1 shows the maximum fluorescence excitation and emission peaks of the prepared CuNCs. The diluted yellowand blue-emitting CuNC solutions were nearly colorless (or very pale yellow) and transparent under visible light, whereas they exhibited yellow and blue fluorescence under UV light irradiation $(365 \mathrm{~nm})$, respectively (inset of Figure 1$)$. As shown in Figure S1A, the emission wavelength of blue-emitting CuNCs was red-shifted from 415 to $475 \mathrm{~nm}$ with the excitation wavelength ranging from 310 to $400 \mathrm{~nm}$, whereas the emission wavelength was almost independent of the excitation wavelength for the yellow-emitting CuNCs (Figure S1B). The difference of the fluorescence behaviors may be caused by the different surface states of the CuNCs with blue and yellow emission. The absolute quantum yields (QYs) for the CuNCs in aqueous solutions were measured as 3.1 and $0.1 \%$ for yellow and blue emission, respectively. The morphology and size of CuNCs were clearly revealed by transmission electron microscopy (TEM) images. Figure 2 shows that CuNCs were highly uniform and monodisperse. The average diameters of CuNCs for blue and yellow emission were about 1.8 and 2.5 $\mathrm{nm}$, respectively. These results were highly in accord with the phenomenon of fluorescence wavelength dependence on the size of $\mathrm{CuNCs}$. That is, the larger size of CuNCs corresponded to the red-shifted fluorescence emission wavelength, similar to that for other fluorescent nanostructures such as AuNCs. ${ }^{36}$ Figure S2 shows the UV-vis absorption spectra of the asprepared CuNCs and trypsin. The absorption spectrum of trypsin had a peak centered at $276 \mathrm{~nm}$, and it was changed when $\mathrm{CuNCs}$ were formed. The rather broad spectra with a small red-shifted hump confirmed the formation of CuNCs. Furthermore, there was no apparent surface plasmon resonance absorption band appearing at around $560-600 \mathrm{~nm}$, indicating no large copper nanoparticles in the CuNC samples. ${ }^{37,38}$ The slight blue shift of the absorption for the blue-emitting CuNCs compared to that of the yellow-emitting CuNCs further confirmed that the blue-emitting CuNCs were smaller in size than that of the yellow-emitting CuNCs. ${ }^{39}$

In most reports, MNCs synthesized with the assistance of proteins were generally prepared under basic $\mathrm{pH}$ conditions. $^{40-44}$ It was mainly because proteins possessed good reducing capacity when the reaction $\mathrm{pH}$ was greater than $\mathrm{p} K_{\mathrm{a}}$ of Tyr $(\sim 10)^{44}$ and it could act as a reductant. However, in the present study, yellow-emitting CuNCs were obtained when the synthesis was conducted at $\mathrm{pH}$ 3.4. Although it was unclear how trypsin "biomineralized" fluorescent CuNCs, the present results clearly showed that the $\mathrm{pH}$ of the reaction solution played an important role in the determination of CuNCs with yellow and blue fluorescent emission. Far-UV circular dichroism (CD) spectra of the trypsin solution at different $\mathrm{pH}$ values were recorded to investigate the mechanism of $\mathrm{pH}$ dependent formation of multicolored CuNCs. As shown in Figure 3, compared to that for the trypsin at $\mathrm{pH} 12.0$, a negative band at around $198 \mathrm{~nm}$ from the random coil became more predominant for the trypsin at $\mathrm{pH}$ 3.4. It indicated that a large conformational change for trypsin occurred because of the denaturation of trypsin under acidic $\mathrm{pH}$ conditions. More

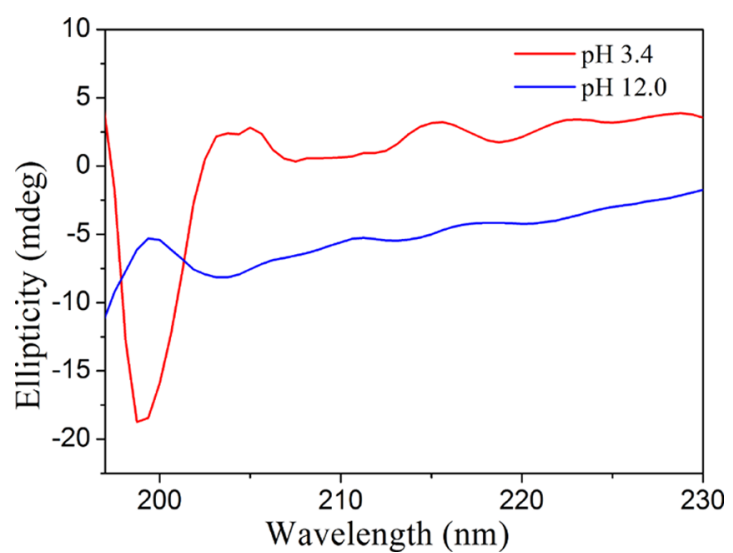

Figure 3. CD spectra of the aqueous solution of trypsin at different pHs.

functional groups, such as $-\mathrm{OH},-\mathrm{NH}$, and $-\mathrm{COOH}$, could thus be accessible to interact with copper, and large internal spaces within the weak-bonding random-coiled trypsin may be utilized for the formation of large CuNCs. Accordingly, it was concluded that different secondary structures of trypsin at different $\mathrm{pH}$ levels could affect the formation of CuNCs with different sizes. Fourier transform infrared (FT-IR) spectra is an excellent tool for the structural characterization of proteins in various environments. It has been reported that the amide I band in the FT-IR spectrum was also sensitive to the change of protein secondary structure. ${ }^{45}$ Figure S3 revealed that the characteristic vibration peaks of trypsin were unaltered after the blue-emitting CuNCs formed, whereas an obvious shift of the amide I band to lower wavenumbers was observed after the formation of yellow-emitting CuNCs. This indicated that a conformational change for the trypsin of yellow-emitting CuNCs occurred under acidic $\mathrm{pH}$ conditions. Therefore, all of these results confirmed that the mechanism for the formation of $\mathrm{pH}$-dependent multicolored $\mathrm{CuNCs}$ was based on the changes of the secondary structure of trypsin at different $\mathrm{pH}$ levels.

In the present study, as the yellow-emitting $\mathrm{CuNCs}$ possessed good water solubility and strong fluorescence intensity, they could be explored as a fluorescent probe for practical sensing. To improve the sensitivity of the fluorescent probe, several experimental conditions including the concentration, temperature, and reaction time were optimized to obtain yellow-emitting CuNCs with high fluorescence intensity. In this method, we found that $\mathrm{N}_{2} \mathrm{H}_{4}$ was necessary in the preparation of CuNCs. As shown in Figure S4, in the preparation of yellow-emitting CuNCs, the product synthesized in the absence of $\mathrm{N}_{2} \mathrm{H}_{4}$ exhibited no fluorescence signal. From this phenomenon, it could be concluded that trypsin alone was not enough to reduce $\mathrm{Cu}$ ions. $\mathrm{N}_{2} \mathrm{H}_{4}$ was deemed as a reducing agent. In addition to $\mathrm{N}_{2} \mathrm{H}_{4}$, several other reducing agents such as ascorbate and $\mathrm{NaBH}_{4}$ were also applied for the synthesis of yellow-emitting CuNCs. As shown in Figure S5, the CuNCs with $\mathrm{N}_{2} \mathrm{H}_{4}$ as the reducing agent exhibited relatively strong fluorescence intensity. The fluorescence spectra of the CuNCs prepared with different molar ratios of $\mathrm{CuSO}_{4}$ and $\mathrm{N}_{2} \mathrm{H}_{4}$ (keeping the concentration of trypsin constant) are shown in Figure S6A. It could be seen that the product with a molar ratio of 1:1 exhibited the maximum fluorescence intensity at $567 \mathrm{~nm}$. The reaction temperature was also investigated in the synthesis of fluorescent CuNCs. As shown in Figure S6B, external heat 

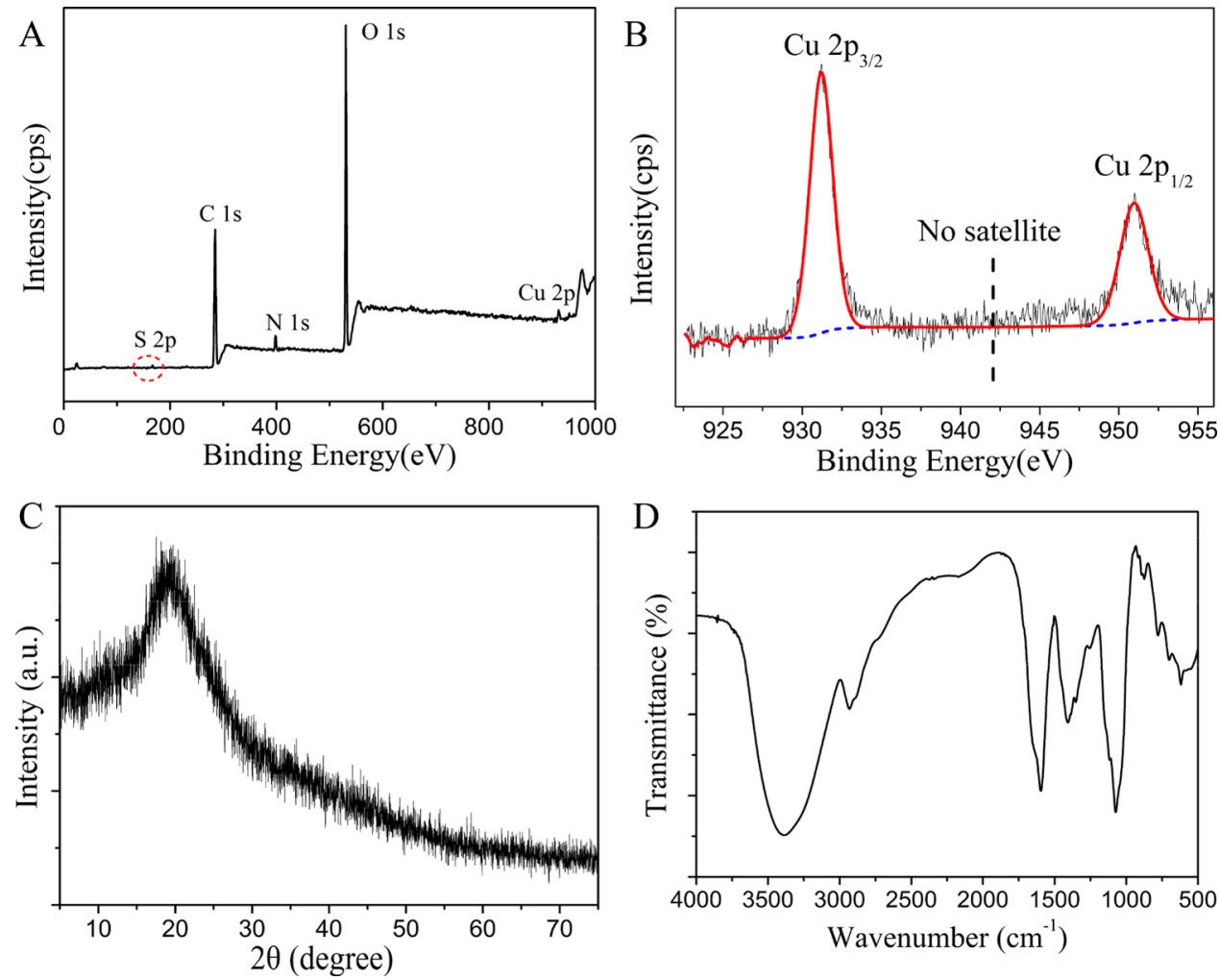

Figure 4. (A) XPS full-scan spectrum of CuNCs. (B) High-resolution XPS spectrum of the Cu 2p peak of CuNCs. (C) Powder XRD pattern of CuNCs. (D) FT-IR spectrum of yellow-emitting CuNCs.
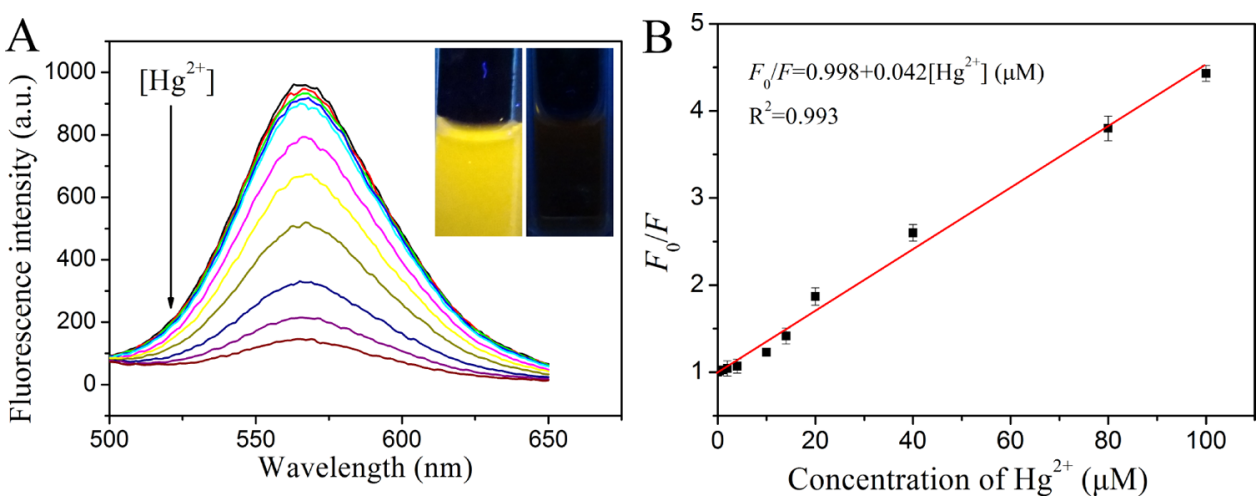

Figure 5. (A) Fluorescence emission spectra of CuNCs upon addition of various concentrations of $\mathrm{Hg}^{2+}$. The insets show the photographs of $\mathrm{CuNC}$ solutions in the absence and presence of $100 \mu \mathrm{M} \mathrm{Hg}^{2+}$ under UV light $(365 \mathrm{~nm})$. (B) Relationship between $F_{0} / F$ and the concentration of $\mathrm{Hg}^{2+}$ in the range of $0.1-100 \mu \mathrm{M}$.

could significantly accelerate the generation of CuNCs; thus, 70 ${ }^{\circ} \mathrm{C}$ was chosen as the reaction temperature. Under these reaction conditions, the fluorescence intensity reached maximum with the reaction time up to $2.0 \mathrm{~h}$, and after that, it decreased (Figure S6C). This result might be attributed to the redistribution or interprotein transfer of copper ions after $2.0 \mathrm{~h}^{46}$ Therefore, an optimum reaction time of $2.0 \mathrm{~h}$ was used in the whole study. Therefore, the yellow-emitting CuNCs prepared under optimal synthetic conditions were used for conducting the following research.

It was well known that $\mathrm{Cu}$ was easily oxidized because of its low reduction potential. Therefore, it was important to confirm the oxidation state of $\mathrm{Cu}$ in the CuNC sample. An X-ray photoelectron spectroscopy (XPS) survey spectrum showed that the sample was composed of all of the expected elements
$\mathrm{C}, \mathrm{N}, \mathrm{O}, \mathrm{S}$, and $\mathrm{Cu}$ (Figure 4A). The high-resolution XPS spectrum of the $\mathrm{Cu} 2 \mathrm{p}$ peak of CuNCs is displayed in Figure 4B. Two intense peaks at 951.0 and $931.2 \mathrm{eV}$ were assigned to the binding energies of $\mathrm{Cu} 2 \mathrm{p}_{1 / 2}$ and $2 \mathrm{p}_{3 / 2}$ from $\mathrm{Cu}(0)$, and the result was consistent with the previous report. ${ }^{9,41}$ In addition, no characteristic satellite peak at around $942 \mathrm{eV}$ implied the absence of $\mathrm{Cu}^{2+}$ in CuNCs. This thus precluded any significant oxidation of CuNCs. ${ }^{47}$ Nevertheless, it was known that the typical $2 \mathrm{p}_{3 / 2}$ binding energy of $\mathrm{Cu}(0)$ was only $\sim 0.1 \mathrm{eV}$ away from that of the $\mathrm{Cu}(\mathrm{I})$ species. $^{8}$ Therefore, the valence state of $\mathrm{Cu}$ in our samples likely lied between 0 and +1 . The powder $\mathrm{X}$ ray diffraction (XRD) pattern of CuNCs showed a broad peak at around $20^{\circ}$ (Figure 4C). The result supported the absence of a significant population of crystalline $\mathrm{Cu}$ nanoparticles in the sample. ${ }^{48}$ Next, the surface bonds of the synthesized CuNCs 
Table 1. Comparison of the Sensing Performance of Different Fluorescent Probes for $\mathbf{H g}^{2+}$ Detection

\begin{tabular}{lllll}
\multicolumn{1}{c}{ sensing material } & linear range $(\mu \mathrm{M})$ & LOD $(\mathrm{nM})$ & response time $(\mathrm{min})$ & reference \\
nitrogen-doped carbon quantum dots & $0-25$ & 230 & & 49 \\
trypsin-stabilized AuNCs & $0.05-0.6$ & 50 & & 50 \\
$\beta$-lactoglobulin-stabilized AuNCs & $0.05-500$ & 30 & 5 & 51 \\
BSA-AuAg BNCs & $0.05-6.3$ & 13 & $>60$ & 52 \\
DNA duplex-templated AgNCs & $0.01-0.3$ & 10 & 3 & 53 \\
oligonucleotide-stabilized AgNCs & $0.005-1.5$ & 5 & 30 & 54 \\
DNA-templated AgNCs & $0.0025-0.05$ & 0.9 & 2 & 55 \\
CuNCs & $0.1-100$ & 30 & & this work \\
\hline
\end{tabular}
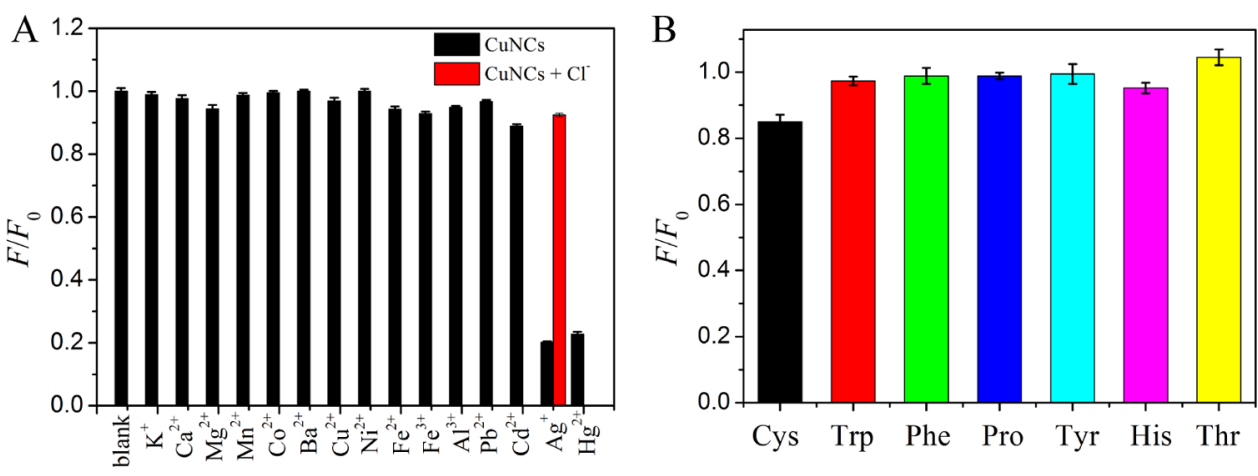

Figure 6. Selectivity of the CuNC sensor toward $\mathrm{Hg}^{2+}$ over other metal ions (A) and amino acids (B). The concentrations of $\mathrm{Hg}^{2+}$ and interfering substances were 100 and $500 \mu \mathrm{M}$, respectively. The concentration of $\mathrm{Cl}^{-}$was $2 \mathrm{mM}$.

were analyzed by FT-IR. As shown in Figure 4D, the peaks at $3400-3000 \mathrm{~cm}^{-1}$ due to $-\mathrm{NH}$ and $-\mathrm{OH}$ stretching vibrations were also prominent in the spectra, indicating the existence of free $-\mathrm{NH}_{2} /-\mathrm{COOH}$ groups in CuNCs.

To test the feasibility of using the as-prepared CuNCs in practical sensing applications, the stability of the CuNC probe was investigated. As shown in Figure S7, both the blue- and yellow-emitting CuNCs were observed to be very stable that the fluorescence intensity had no change under continuous light irradiation for $60 \mathrm{~min}$. In addition, CuNCs displayed relatively stable fluorescence intensities even under extreme $\mathrm{pH}$ conditions (Figure S8). From Figure S9, it could be noted that the fluorescence intensity remained nearly constant when the concentration of $\mathrm{NaCl}$ was as high as $50 \mathrm{mM}$. These results indicated that the as-prepared CuNCs had excellent photostability and salt tolerance.

In this work, it was observed that the fluorescence intensity of the yellow-emitting CuNCs was sensitively quenched in the presence of $\mathrm{Hg}^{2+}$. As demonstrated in Figure 5A, with the addition of different concentrations of $\mathrm{Hg}^{2+}$, the fluorescence intensity of the CuNC solution decreased proportionately. The fluorescence response was rapid, and the reaction completely achieved a balance within $1 \mathrm{~min}$ (Figure S10). To achieve maximum quenching efficiency, the type of buffer solution and detection $\mathrm{pH}$ value have been optimized. As shown in Figure S11A, CuNCs exhibited strongest fluorescence intensity in the pH 4.0 phosphate-buffered saline (PBS) buffer solution compared to that in other buffer solutions. In addition, with the addition of $\mathrm{Hg}^{2+}$ to the $\mathrm{CuNC}$ solution, the fluorescence quenching efficiency reached maximum in the $\mathrm{pH}$ 4.0 PBS buffer solution (Figure S11B). Therefore, the $\mathrm{pH} 4.0$ PBS buffer solution was selected for detection of $\mathrm{Hg}^{2+}$. Under optimum conditions, the quenching efficiency $\left(F_{0} / F\right)$ displayed a good linear relationship $\left(R^{2}=0.993\right)$ with the concentration of $\mathrm{Hg}^{2+}$ ranging from 0.1 to $100 \mu \mathrm{M}$, where $F_{0}$ and $F$ are the fluorescence intensities of the $\mathrm{CuNC}$ solution in the absence and presence of $\mathrm{Hg}^{2+}$, respectively. The limit of detection (LOD) $(3 s / k$, in which $s$ is the standard deviation for the control and $k$ is the slope of the calibration curve) was estimated to be $30 \mathrm{nM}$, which was lower or comparable to that obtained by other fluorescent probes for $\mathrm{Hg}^{2+}$ sensing (Table 1). ${ }^{49-52}$ It should be noted that the sensitivity of the CuNC sensor for $\mathrm{Hg}^{2+}$ was lower than that of DNA-templated fluorescence nanoclusters. ${ }^{53-55}$ Nevertheless, the proposed method in this work was much easy-going and time-saving, which made it more convenient for practical applications.

Besides those of $\mathrm{Hg}^{2+}$, the effects of some other metal ions and several amino acids on the assay system were further investigated under the same test conditions. As shown in Figure 6 , the fluorescence intensity of CuNCs decreased significantly by adding $\mathrm{Hg}^{2+}$ to the solution, whereas other metal ions $\left(\mathrm{K}^{+}\right.$, $\mathrm{Ca}^{2+}, \mathrm{Mg}^{2+}, \mathrm{Mn}^{2+}, \mathrm{Co}^{2+}, \mathrm{Ba}^{2+}, \mathrm{Cu}^{2+}, \mathrm{Ni}^{2+}, \mathrm{Fe}^{2+}, \mathrm{Fe}^{3+}, \mathrm{Al}^{3+}, \mathrm{Cd}^{2+}$, and $\mathrm{Pb}^{2+}$ ) and several amino acids (Cys, Trp, Pro, Tyr, His, Thr, Phe) had only a slight or negligible effect on the fluorescence intensity, even when the concentration of the potential interferences was 5 -fold higher than that of $\mathrm{Hg}^{2+}$. It is worth mentioning that other than $\mathrm{Hg}^{2+}, \mathrm{Ag}^{+}$ions also led to great decreases in the fluorescence intensity. To eliminate the interference, a chelating ligand, sodium chloride $(2.0 \mathrm{mM})$, which showed effective masking ability for $\mathrm{Ag}^{+}$, was added to the solution. As a result, even in the presence of $\mathrm{Ag}^{+}$at a concentration 5 times greater than that of $\mathrm{Hg}^{2+}$, no obvious fluorescence quenching was observed, thus exhibiting improved selectivity of the CuNC probe toward $\mathrm{Hg}^{2+}$. The results demonstrated that the fluorescent $\mathrm{CuNC}$ probe exhibited excellent selectivity toward $\mathrm{Hg}^{2+}$.

To date, several $\mathrm{Hg}^{2+}$-induced fluorescence quenching mechanisms have been proposed. Morishita et al. noticed a significant quenching of $\mathrm{AgNCs}$ by $\mathrm{Hg}^{2+}$, and they attributed it to the redox reaction mechanism. ${ }^{56}$ In our present work, the 

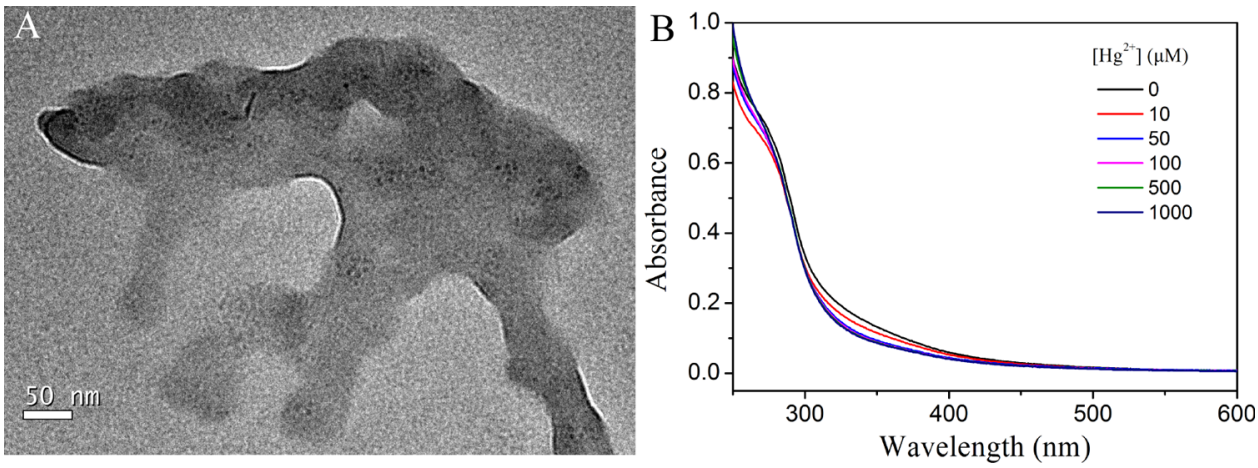

Figure 7. (A) TEM image of CuNCs after addition of $\mathrm{Hg}^{2+}$. (B) UV-vis spectra of CuNCs before and after addition of different concentrations of $\mathrm{Hg}^{2+}$.

oxidation state of $\mathrm{Cu}$ in CuNCs was investigated by the XPS spectra in the absence and presence of $100 \mu \mathrm{M} \mathrm{Hg}{ }^{2+}$, respectively. As shown in Figure S12, the addition of $\mathrm{Hg}^{2+}$ to the $\mathrm{CuNC}$ solution had little effect on the oxidation state of $\mathrm{Cu}$, which ruled out the redox-reaction-induced $\mathrm{CuNC}$ fluorescence quenching. Other fluorescence quenching mechanisms could be taken into consideration. To study the $\mathrm{Hg}^{2+}$-induced fluorescence quenching mechanism, the TEM image of CuNCs after addition of $\mathrm{Hg}^{2+}$ was investigated (Figure 7A). It was clear that $\mathrm{CuNCs}$ obviously aggregated after $\mathrm{Hg}^{2+}$ was added. As it was reported, $\mathrm{Hg}^{2+}$ has a strong affinity toward amino and carboxylic groups on the surface of CuNCs. ${ }^{57,58}$ The interaction between $\mathrm{Hg}^{2+}$ and CuNCs made the CuNCs close to each other. Thus, fluorescence quenching of CuNCs was ascribed to the aggregation of CuNCs induced by $\mathrm{Hg}^{2+}$, thus facilitating the efficient energy transfer. The phenomenon was consistent with the previous report by Huang. ${ }^{59}$ In addition, the fact that quenching by $\mathrm{Hg}^{2+}$ did not affect markedly either the fluorescence emission spectrum or the absorption spectrum of CuNCs (Figure 7B) further indicated the quenching mechanism of energy transfer between $\mathrm{CuNCs}$ and $\mathrm{Hg}^{2+} \cdot 60$

The practical application of this fluorescence method was evaluated through the detection of $\mathrm{Hg}^{2+}$ in human urine and serum samples. Three concentrations of $\mathrm{Hg}^{2+}$ were spiked into the samples. The recovery values were in the range of $89.0-$ 105.0 and $95.0-108.8 \%$ in urine and serum samples, respectively (Table 2). These results demonstrated that the current strategy for $\mathrm{Hg}^{2+}$ sensing in practical samples was reliable and feasible.

Table 2. Analytical Results for the Detection of $\mathrm{Hg}^{2+}$ Ions in Different Natural Samples by the Proposed Method ${ }^{a}$

\begin{tabular}{crrrrr} 
& \multicolumn{2}{c}{ urine } & & \multicolumn{2}{c}{ serum } \\
\cline { 2 - 3 } \cline { 5 - 6 } $\begin{array}{c}\text { spiked concentration } \\
(\mu \mathrm{M})\end{array}$ & found $(\mu \mathrm{M})$ & $\begin{array}{c}\text { recovery } \\
(\%)\end{array}$ & & found $(\mu \mathrm{M})$ & $\begin{array}{c}\text { recovery } \\
(\%)\end{array}$ \\
4.0 & $4.2 \pm 0.5$ & 105.0 & & $3.8 \pm 0.4$ & 95.0 \\
20.0 & $17.8 \pm 0.4$ & 89.0 & & $20.1 \pm 0.8$ & 100.5 \\
40.0 & $37.3 \pm 0.4$ & 93.3 & & $43.5 \pm 0.3$ & 108.8 \\
$a_{n=3 .}$ & & & & \\
\hline
\end{tabular}

To apply the yellow-emitting CuNCs in the field of biological imaging, 3-(4,5-dimethylthiazol-2-yl)-2,5-diphenyltetrazolium bromide (MTT) assays were carried out to assess the cytotoxicity of the CuNC probes to HeLa cells. HeLa cells were incubated with various concentrations of CuNCs in standard cell culture conditions. After incubation for $24 \mathrm{~h}$, the viability of the cells was determined. As shown in Figure 8, the cell viability was found to be greater than $82 \%$ even when the

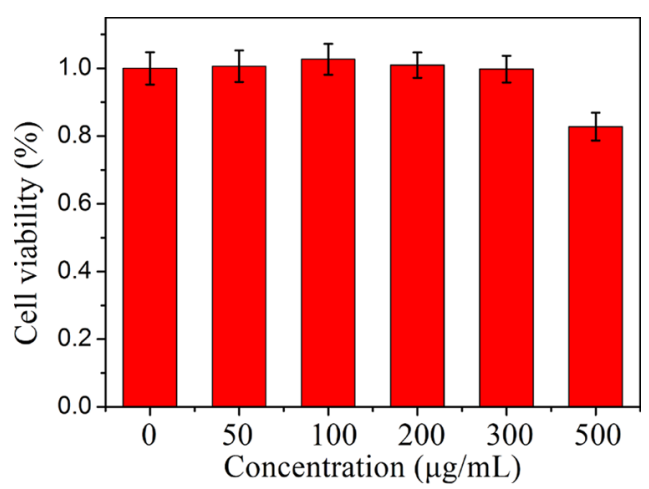

Figure 8. Cell viability of HeLa cells in the presence of different concentrations of CuNCs.

concentration of CuNCs was up to $500 \mu \mathrm{g} / \mathrm{mL}$. High cell viability demonstrated the low toxicity and excellent biocompatibility of the as-prepared CuNCs, which made them suitable for cell imaging.

As shown in Figure 9, by incubating Hela cells with CuNCs $(500 \mu \mathrm{g} / \mathrm{mL})$ for $1 \mathrm{~h}$ at $37{ }^{\circ} \mathrm{C}$, a significant yellow emission from the intracellular region could be observed. All of these results showed that the yellow-emitting CuNCs could be applied in the field of biological imaging and cell labeling.

\section{CONCLUSIONS}

In summary, CuNCs with yellow and blue fluorescent emission were synthesized with a facile approach in the presence of trypsin and $\mathrm{N}_{2} \mathrm{H}_{4}$. The $\mathrm{pH}$ of the reaction solution was critical in determining whether CuNCs showed yellow or blue fluorescent emission. As the yellow-emitting CuNCs exhibited excellent stability, low toxicity, and good biocompatibility, the fluorescent CuNCs were successfully used in not only the detection of $\mathrm{Hg}^{2+}$ but also cell imaging in $\mathrm{HeLa}$ cells. Therefore, this facile preparation of multicolored CuNCs offered access to promising candidates for biological labeling and sensing applications.

\section{EXPERIMENTAL SECTION}

Materials. Trypsin from bovine pancreas was obtained from Aladdin Co., Ltd (Shanghai, China). $\mathrm{CuSO}_{4} \cdot 5 \mathrm{H}_{2} \mathrm{O}$ was purchased from Shanghai Bodi Chemical Co., Ltd (Shanghai, China). $\mathrm{HgCl}_{2}, \mathrm{KCl}, \mathrm{CaCl}_{2}, \mathrm{MgCl}_{2}, \mathrm{MnCl}_{2}, \mathrm{CoCl}_{2}, \mathrm{BaCl}_{2}$, 


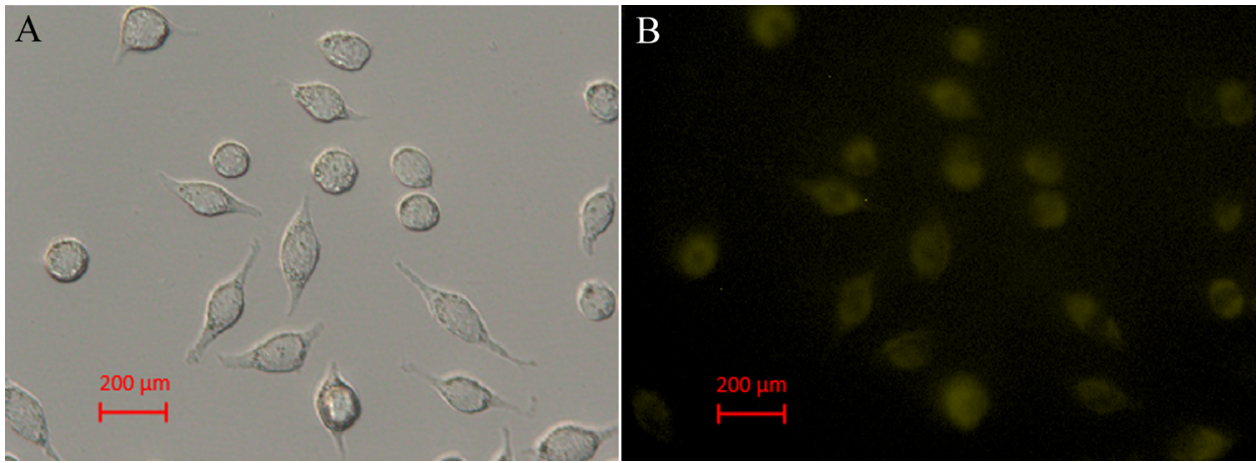

Figure 9. Fluorescence microscopy image (A) and its corresponding bright-field transmission image (B) of HeLa cells incubated with $500 \mu \mathrm{g} / \mathrm{mL}$ CuNCs for $1 \mathrm{~h}$ at $37^{\circ} \mathrm{C}$.

$\mathrm{CuCl}_{2}, \mathrm{NiCl}_{2}, \mathrm{FeCl}_{2}, \mathrm{FeCl}_{3}$, and $\mathrm{AlCl}_{3}$ were purchased from Sinopharm Chemical Reagent Co., Ltd. (Shanghai, China). LTryptophan (L-Trp), L-proline (L-Pro), L-tyrosine (L-Tyr), Lhistidine (L-His), L-threonine (L-Thr), and L-phenylalanine ( $\mathrm{L}$ Phe) were purchased from Shanghai Sangon Biotechnology Co., Ltd. (Shanghai, China).

pH-Dependent Synthesis of Copper Nanoclusters (CuNCs). All glassware was cleaned in a bath of freshly prepared 3:1 $\mathrm{HCl} / \mathrm{HNO}_{3}$ and rinsed thoroughly in water before use. Yellow-emitting CuNCs were prepared as follows. Typically, $1 \mathrm{~mL}$ of $\mathrm{CuSO}_{4}$ solution $(10 \mathrm{mM})$ was added to 1 $\mathrm{mL}$ of trypsin $(40 \mathrm{mg} / \mathrm{mL})$ under vigorous stirring at room temperature. Five minutes later, the $\mathrm{pH}$ of the obtained solution was about 3.4. Then, $100 \mu \mathrm{L}$ of $\mathrm{N}_{2} \mathrm{H}_{4}$ solution (100 $\mathrm{mM}$ ) was added dropwise under vigorous stirring. The reaction mixture was incubated at $70{ }^{\circ} \mathrm{C}$ in a water bath for $2 \mathrm{~h}$, and the color changed gradually from light blue to pale yellow. After the reaction, $\mathrm{CuNCs}$ were purified by centrifuging at $12000 \mathrm{rpm}$ to remove large particles. The resultant yellow-emitting $\mathrm{CuNCs}$ were stored at $4{ }^{\circ} \mathrm{C}$ for further use.

To obtain the blue-emitting CuNCs, similar synthesis was conducted except that the $\mathrm{pH}$ of the solution before addition of $\mathrm{N}_{2} \mathrm{H}_{4}$ was adjusted to 12 by $1 \mathrm{M} \mathrm{NaOH}$. The final dark brown solution of CuNCs exhibited a blue-emitting fluorescence under UV lamp irradiation.

Fluorescence Detection of $\mathbf{H g}^{2+}$. For the typical assay of $\mathrm{Hg}^{2+}, 300 \mu \mathrm{L}$ of the prepared yellow-emitting CuNCs solution was added into $2.2 \mathrm{~mL}$ of the PBS buffer solution ( $\mathrm{pH} 4.0,10.0$ $\mathrm{mM})$ to prepare the probe solution. The solution $(10.0 \mu \mathrm{L})$ with different concentrations of $\mathrm{Hg}^{2+}$ was added into the probe solution. Fluorescence emission spectra were collected with excitation at $360 \mathrm{~nm}$ after $60 \mathrm{~s}$. In the selectivity experiment, a series of potential metal ions and amino acids were mixed with the probe solution. The concentrations of the these interferences were $500 \mu \mathrm{M}$.

Characterization. All of the instruments used for characterization were the same as those used in the previous work. ${ }^{10,61}$ Transmission electron microscopy (TEM) images of CuNCs with different fluorescent emission were obtained using a Tecnai G2F30 instrument. Fourier transform infrared (FT-IR) spectra were recorded on a Nicolet Nexus 670 spectrometer using $\mathrm{KBr}$ pellets. Powder X-ray diffraction (XRD) patterns were recorded on a $\mathrm{D} / \max 82400 \mathrm{X}$-ray powder diffractometer (Rigaku, Japan) with $\mathrm{Cu} \mathrm{K} \alpha$ radiation $(\lambda=0.154056 \AA)$. X-ray photoelectron spectroscopy (XPS) measurement was performed using a PerkinElmer PHI-5702 multifunctional photoelectron spectrometer equipped with an $\mathrm{Al} \mathrm{K} \alpha$ exciting source.
Far-UV circular dichroism (CD) spectra of trypsin under different $\mathrm{pH}$ conditions were recorded at $25{ }^{\circ} \mathrm{C}$ on an Olis DSM 1000 double-beam spectrophotometer. UV-visible absorption spectra were recorded by a TU-1901 double-beam UV-vis spectrophotometer. Fluorescence measurements were carried out using a RF-5301 spectrofluorophotometer with both excitation and emission slits set at $10.0 \mathrm{~nm}$. The excitation wavelength was set at $360 \mathrm{~nm}$. Samples for absorption and emission measurements were taken in $1 \mathrm{~cm} \times 1 \mathrm{~cm}$ quartz cuvette. The absolute photoluminescence quantum yield (QY) of CuNCs was measured and calculated using an "Edinburgh Instruments" FLS 920 spectrometer, which has been reported by our previous work (see Supporting Information)..$^{10}$

MTT Assay. The human cervical carcinoma HeLa cells were cultured in RPMI-1640 medium supplemented with $10 \%$ fetal bovine serum using a 96-well culture plate and kept in an incubator at $37{ }^{\circ} \mathrm{C}$ with a humidified atmosphere of $5 \% \mathrm{CO}_{2}$. Prior to test, $1 \times 10^{4}$ cells were incubated in 96-well plates for $24 \mathrm{~h}$ at $37{ }^{\circ} \mathrm{C}$ in a final volume of $100 \mu \mathrm{L}$. Then, $10 \mu \mathrm{L}$ of CuNCs with different concentrations $(0,50,100,200,300$, and $500 \mu \mathrm{g} / \mathrm{mL}$, respectively) was added and incubated for another $24 \mathrm{~h}$. Afterward, cells were rinsed twice with PBS (10 mM, pH 7.4) followed by addition of $100 \mu \mathrm{L}$ of fresh medium and $10 \mu \mathrm{L}$ of 3-(4,5-dimethylthiazol-2-yl)-2,5-diphenyltetrazolium bromide (MTT) $(5 \mathrm{mg} / \mathrm{mL})$ to each well. The cells were incubated for additional $4 \mathrm{~h}$ at $37^{\circ} \mathrm{C}$. After removing all medium from the wells, $100 \mu \mathrm{L}$ of dimethylsulfoxide was added to each well and mixed thoroughly for $5 \mathrm{~min}$. The optical density (OD) of the mixture was measured at $570 \mathrm{~nm}$ using a microplate reader. The cell viability was estimated as (OD treated/OD control) $\times 100 \%$, where OD control and OD treated were obtained in the absence and presence of CuNCs, respectively. ${ }^{62}$

In Vivo Fluorescence Imaging. The HeLa cells were cultured in RPMI-1640 medium supplemented with $10 \%$ fetal bovine serum at $37{ }^{\circ} \mathrm{C}$ with $5 \% \mathrm{CO}_{2}$ overnight. Then, $\mathrm{CuNCs}$ $(500 \mu \mathrm{g} / \mathrm{mL})$ were added to the cell culture, and the cells were incubated for another $1 \mathrm{~h}$ at $37^{\circ} \mathrm{C}$. After the cells were washed with PBS three times, the fluorescence images were acquired by a fluorescent microscope.

\section{ASSOCIATED CONTENT}

S Supporting Information

The Supporting Information is available free of charge on the ACS Publications website at DOI: 10.1021/acsomega.7b01052.

Details about the absolute QY and additional Figures (S1-S12) (PDF) 


\section{AUTHOR INFORMATION}

\section{Corresponding Authors}

*E-mail: chyl@lzu.edu.cn. Tel: +86-931-8912763. Fax: +86931-8912582 (Y.C.).

*E-mail: chenxg@lzu.edu.cn (X.C.).

\section{ORCID}

Xingguo Chen: 0000-0002-7982-1519

\section{Notes}

The authors declare no competing financial interest.

\section{ACKNOWLEDGMENTS}

The authors are grateful for financial support from the National Nature Science Foundation of China (No. 21675068).

\section{REFERENCES}

(1) Gittins, D. I.; Bethell, D.; Schiffrin, D. J.; Nichols, R. J. A nanometre-scale electronic switch consisting of a metal cluster and redox-addressable groups. Nature 2000, 408, 67-69.

(2) Luo, Z.; Zheng, K.; Xie, J. Engineering ultrasmall water-soluble gold and silver nanoclusters for biomedical applications. Chem. Commun. 2014, 50, 5143-5155.

(3) Zhang, L.; Wang, E. Metal nanoclusters: New fluorescent probes for sensors and bioimaging. Nano Today 2014, 9, 132-157.

(4) Shojaeifard, Z.; Hemmateenejad, B.; Shamsipur, M. Efficient onoff ratiometric fluorescence probe for cyanide ion based on perturbation of the interaction between gold nanoclusters and a copper(II)-phthalocyanine complex. ACS Appl. Mater. Interfaces 2016, $8,15177-15186$.

(5) Wang, C.; Lin, H.; Xu, Z.; Huang, Y.; Humphrey, M. G.; Zhang, C. Tunable carbon-dot-based dual-emission fluorescent nanohybrids for ratiometric optical thermometry in living cells. ACS Appl. Mater. Interfaces 2016, 8, 6621-6628.

(6) Venkatesh, V.; Shukla, A.; Sivakumar, S.; Verma, S. Purinestabilized green fluorescent gold nanoclusters for cell nuclei imaging applications. ACS Appl. Mater. Interfaces 2014, 6, 2185-2191.

(7) Xu, Y.-K.; Hwang, S.; Kim, S.; Chen, J.-Y. Two orders of magnitude fluorescence Enhancement of aluminum phthalocyanines by gold nanocubes: a remarkable improvement for cancer cell imaging and detection. ACS Appl. Mater. Interfaces 2014, 6, 5619-5628.

(8) Wei, W.; Lu, Y.; Chen, W.; Chen, S. One-pot synthesis, photoluminescence, and electrocatalytic properties of subnanometersized copper clusters. J. Am. Chem. Soc. 2011, 133, 2060-2063.

(9) Jia, X.; Li, J.; Wang, E. Cu nanoclusters with aggregation induced emission enhancement. Small 2013, 9, 3873-3879.

(10) Feng, J.; Ju, Y.; Liu, J.; Zhang, H.; Chen, X. Polyethyleneiminetemplated copper nanoclusters via ascorbic acid reduction approach as ferric ion sensor. Anal. Chim. Acta 2015, 854, 153-160.

(11) Chen, J.; Ji, X.; Tinnefeld, P.; He, Z. Multifunctional dumbbellshaped DNA-templated selective formation of fluorescent silver nanoclusters or copper nanoparticles for sensitive detection of biomolecules. ACS Appl. Mater. Interfaces 2016, 8, 1786-1794.

(12) Huang, H.; Li, H.; Wang, A.-J.; Zhong, S.-X.; Fang, K.-M.; Feng, J.-J. Green synthesis of peptide-templated fluorescent copper nanoclusters for temperature sensing and cellular imaging. Analyst 2014, 139, 6536-6541.

(13) Goswami, N.; Giri, A.; Bootharaju, M. S.; Xavier, P. L.; Pradeep, T.; Pal, S. K. Copper quantum clusters in protein matrix: potential sensor of $\mathrm{Pb}^{2+}$ ion. Anal. Chem. 2011, 83, 9676-9680.

(14) Kawasaki, H.; Hamaguchi, K.; Osaka, I.; Arakawa, R. phdependent synthesis of pepsin-mediated gold nanoclusters with blue green and red fluorescent emission. Adv. Funct. Mater. 2011, 21, $3508-3515$.

(15) Karunasagar, D.; Arunachalam, J.; Gangadharan, S. Development of a 'collect and punch' cold vapour inductively coupled plasma mass spectrometric method for the direct determination of mercury at nanograms per litre levels. J. Anal. At. Spectrom. 1998, 13, 679-682.
(16) Cizdziel, J. V.; Gerstenberger, S. Determination of total mercury in human hair and animal fur by combustion atomic absorption spectrometry. Talanta 2004, 64, 918-921.

(17) Kim, H.-J.; Park, D.-S.; Hyun, M.-H.; Shim, Y.-B. Determination of $\mathrm{HgII}$ ion with a 1,11-Bis(8-quinoyloxy)-3,6,9-trioxaundecanemodified glassy carbon electrode using spin-coating technique. Electroanalysis 1998, 10, 303-306.

(18) Xu, X.; Thundat, T. G.; Brown, G. M.; Ji, H.-F. Detection of $\mathrm{Hg}^{2+}$ using microcantilever sensors. Anal. Chem. 2002, 74, 3611-3615.

(19) Khan, A.; Asiri, A. M.; Khan, A. A. P.; Rub, M. A.; Azum, N.; Rahman, M. M.; Khan, S. B.; Alamry, K. A.; Ghani, S. A. Sol-gel synthesis and characterization of conducting polythiophene/tin phosphate nano tetrapod composite cation-exchanger and its application as $\mathrm{Hg}(\mathrm{II})$ selective membrane electrode. J. Sol-Gel Sci. Technol. 2013, 65, 160-169.

(20) Chen, J.; Zhou, S.; Wen, J. Disposable strip biosensor for visual detection of $\mathrm{Hg}^{2+}$ based on $\mathrm{Hg}^{2+}$-triggered toehold binding and exonuclease III-assisted signal amplification. Anal. Chem. 2014, 86, 3108-3114.

(21) Chen, J.-F.; Han, B.-B.; Ma, J.-F.; Liu, X.; Yang, Q.-Y.; Lin, Q.; Yao, H.; Zhang, Y.-M.; Wei, T.-B. Pillar[5] arene-based fluorescent polymer for selective detection and removal of mercury ions. RSC Adv. 2017, 7, 47709-47714.

(22) Hussain, M. M.; Rahman, M. M.; Arshad, M. N.; Asiri, A. M. $\mathrm{Hg}^{2+}$ sensor development based on (E)-N'-nitrobenzylidene-benzenesulfonohydrazide (NBBSH) derivatives fabricated on a glassy carbon electrode with a nafion matrix. ACS Omega 2017, 2, 420-431.

(23) Arshad, M. N.; Rahman, M. M.; Asiri, A. M.; Sobahi, T. R.; Yu, S.-H. Development of $\mathrm{Hg}^{2+}$ sensor based on $\mathrm{N}$ [prime or minute]-[1(pyridin-2-yl)ethylidene]benzenesulfono-hydrazide (PEBSH) fabricated silver electrode for environmental remediation. RSC Adv. 2015, 5, 81275-81281.

(24) Wen, D.; Deng, L.; Guo, S.; Dong, S. Self-powered sensor for trace $\mathrm{Hg}^{2+}$ detection. Anal. Chem. 2011, 83, 3968-3972.

(25) Zhang, H.-L.; Li, W.-T.; Qu, W.-J.; Wei, T.-B.; Lin, Q.; Zhang, Y.-M.; Yao, H. Mercaptooxazole-phenazine based blue fluorescent sensor for the ultra-sensitive detection of mercury(ii) ions in aqueous solution. RSC Adv. 2017, 7, 47547-47551.

(26) Song, K. C.; Kim, J. S.; Park, S. M.; Chung, K.-C.; Ahn, S.; Chang, S.-K. Fluorogenic $\mathrm{Hg}^{2+}$-selective chemodosimeter derived from 8-hydroxyquinoline. Org. Lett. 2006, 8, 3413-3416.

(27) Ikeda, M.; Matsu-ura, A.; Kuwahara, S.; Lee, S. S.; Habata, Y. $\mathrm{Hg}^{2+}$-sensing system based on structures of complexes. Org. Lett. 2012, $14,1564-1567$.

(28) Li, T.; Dong, S.; Wang, E. Label-free colorimetric detection of aqueous mercury ion $\left(\mathrm{Hg}^{2+}\right)$ using $\mathrm{Hg}^{2+}$-modulated G-quadruplexbased DNAzymes. Anal. Chem. 2009, 81, 2144-2149.

(29) Lin, Q.; Mao, P.-P.; Fan, Y.-Q.; Liu, L.; Liu, J.; Zhang, Y.-M.; Yao, H.; Wei, T.-B. A novel supramolecular polymer gel based on naphthalimide functionalized-pillar[5] arene for the fluorescence detection of $\mathrm{Hg}^{2+}$ and $\mathrm{I}^{-}$and recyclable removal of $\mathrm{Hg}^{2+}$ via cation[small pi] interactions. Soft Matter 2017, 13, 7085-7089.

(30) Chang, H.-C.; Chang, Y.-F.; Fan, N.-C.; Ho, J.-a. A. Facile preparation of high-quantum-yield gold nanoclusters: application to probing mercuric ions and biothiols. ACS Appl. Mater. Interfaces 2014, 6, 18824-18831.

(31) Lu, W.; Qin, X.; Liu, S.; Chang, G.; Zhang, Y.; Luo, Y.; Asiri, A. M.; Al-Youbi, A. O.; Sun, X. Economical, green synthesis of fluorescent carbon nanoparticles and their use as probes for sensitive and selective detection of mercury (II) ions. Anal. Chem. 2012, 84, 5351-5357.

(32) Hu, X.; Wang, W.; Huang, Y. Copper nanocluster-based fluorescent probe for sensitive and selective detection of $\mathrm{Hg}^{2+}$ in water and food stuff. Talanta 2016, 154, 409-415.

(33) Awual, M. R.; Hasan, M. M.; Eldesoky, G. E.; Khaleque, M. A.; Rahman, M. M.; Naushad, M. Facile mercury detection and removal from aqueous media involving ligand impregnated conjugate nanomaterials. Chem. Eng. J. 2016, 290, 243-251.

(34) Li, X.; Zhu, S.; Xu, B.; Ma, K.; Zhang, J.; Yang, B.; Tian, W. Selfassembled graphene quantum dots induced by cytochrome $\mathrm{c}$ : a novel 
biosensor for trypsin with remarkable fluorescence enhancement. Nanoscale 2013, 5, 7776-7779.

(35) Guo, C.; Irudayaraj, J. Fluorescent Ag clusters via a proteindirected approach as a $\mathrm{Hg}(\mathrm{II})$ ion sensor. Anal. Chem. 2011, 83, 2883-2889.

(36) Jiang, H.; Zhang, Y.; Wang, X. Single cytidine units-templated syntheses of multi-colored water-soluble Au nanoclusters. Nanoscale 2014, 6, 10355-10362.

(37) Lisiecki, I.; Pileni, M. Copper metallic particles synthesized "in situ" in reverse micelles: influence of various parameters on the size of the particles. J. Phys. Chem. 1995, 99, 5077-5082.

(38) Mott, D.; Galkowski, J.; Wang, L.; Luo, J.; Zhong, C.-J. Synthesis of size-controlled and shaped copper nanoparticles. Langmuir 2007, 23, 5740-5745.

(39) Shichibu, Y.; Negishi, Y.; Tsunoyama, H.; Kanehara, M.; Teranishi, T.; Tsukuda, T. Extremely high stability of glutathionateprotected Au25 clusters against core etching. Small 2007, 3, 835-839.

(40) Qiao, Y.; Xu, T.; Zhang, Y.; Zhang, C.; Shi, L.; Zhang, G.; Shuang, S.; Dong, C. Green synthesis of fluorescent copper nanoclusters for reversible pH-sensors. Sens. Actuators, B 2015, 220, 1064-1069.

(41) Miao, H.; Zhong, D.; Zhou, Z.; Yang, X. Papain-templated Cu nanoclusters: assaying and exhibiting dramatic antibacterial activity cooperating with $\mathrm{H}_{2} \mathrm{O}_{2}$. Nanoscale 2015, 7, 19066-19072.

(42) Ghosh, S.; Anand, U.; Mukherjee, S. Luminescent silver nanoclusters acting as a label-free photoswitch in metal ion sensing. Anal. Chem. 2014, 86, 3188-3194.

(43) Wang, C.; Wang, C.; Xu, L.; Cheng, H.; Lin, Q.; Zhang, C. Protein-directed synthesis of $\mathrm{pH}$-responsive red fluorescent copper nanoclusters and their applications in cellular imaging and catalysis. Nanoscale 2014, 6, 1775-1781.

(44) Xie, J.; Zheng, Y.; Ying, J. Y. Protein-directed synthesis of highly fluorescent gold nanoclusters. J. Am. Chem. Soc. 2009, 131, 888-889.

(45) Barth, A. Infrared spectroscopy of proteins. Biochim. Biophys. Acta, Bioenerg. 2007, 1767, 1073-1101.

(46) Chaudhari, K.; Xavier, P. L.; Pradeep, T. Understanding the evolution of luminescent gold quantum clusters in protein templates. ACS Nano 2011, 5, 8816-8827.

(47) Koski, K. J.; Cha, J. J.; Reed, B. W.; Wessells, C. D.; Kong, D.; Cui, Y. High-density chemical intercalation of zero-valent copper into $\mathrm{Bi}_{2} \mathrm{Se}_{3}$ nanoribbons. J. Am. Chem. Soc. 2012, 134, 7584-7587.

(48) Ghosh, R.; Sahoo, A. K.; Ghosh, S. S.; Paul, A.; Chattopadhyay, A. Blue-emitting copper nanoclusters synthesized in the presence of lysozyme as candidates for cell labeling. ACS Appl. Mater. Interfaces 2014, 6, 3822-3828.

(49) Zhang, R.; Chen, W. Nitrogen-doped carbon quantum dots: Facile synthesis and application as a "turn-off" fluorescent probe for detection of $\mathrm{Hg}^{2+}$ ions. Biosens. Bioelectron. 2014, 55, 83-90.

(50) Kawasaki, H.; Yoshimura, K.; Hamaguchi, K.; Arakawa, R. Trypsin-stabilized fluorescent gold nanocluster for sensitive and selective $\mathrm{Hg}^{2+}$ detection. Anal. Sci. 2011, 27, 591.

(51) Zang, J.; Li, C.; Zhou, K.; Dong, H.; Chen, B.; Wang, F.; Zhao, G. Nanomolar $\mathrm{Hg}^{2+}$ detection using $\beta$-lactoglobulin-stabilized fluorescent gold nanoclusters in beverage and biological media. Anal. Chem. 2016, 88, 10275-10283.

(52) Zheng, B.; Zheng, J.; Yu, T.; Sang, A.; Du, J.; Guo, Y.; Xiao, D.; Choi, M. M. F. Fast microwave-assisted synthesis of AuAg bimetallic nanoclusters with strong yellow emission and their response to mercury(II) ions. Sens. Actuators, B 2015, 221, 386-392.

(53) Deng, L.; Zhou, Z.; Li, J.; Li, T.; Dong, S. Fluorescent silver nanoclusters in hybridized DNA duplexes for the turn-on detection of $\mathrm{Hg}^{2+}$ ions. Chem. Commun. 2011, 47, 11065-11067.

(54) Guo, W.; Yuan, J.; Wang, E. Oligonucleotide-stabilized Ag nanoclusters as novel fluorescence probes for the highly selective and sensitive detection of the $\mathrm{Hg}^{2+}$ ion. Chem. Commun. 2009, 3395-3397.

(55) Lan, G.-Y.; Chen, W.-Y.; Chang, H.-T. Control of synthesis and optical properties of DNA templated silver nanoclusters by varying DNA length and sequence. RSC $A d v$. 2011, 1, 802-807.
(56) Morishita, K.; MacLean, J. L.; Liu, B.; Jiang, H.; Liu, J. Correlation of photobleaching, oxidation and metal induced fluorescence quenching of DNA-templated silver nanoclusters. Nanoscale 2013, 5, 2840-2849.

(57) Guo, Y.; Wang, Z.; Shao, H.; Jiang, X. Hydrothermal synthesis of highly fluorescent carbon nanoparticles from sodium citrate and their use for the detection of mercury ions. Carbon 2013, 52, 583-589.

(58) Liu, J.; Chen, Y.; Wang, W.; Feng, J.; Peng, S.; Ma, S.; Chen, H.; Chen, $\mathrm{X}$. Effective synthesis of highly fluorescent nitrogen doped carbon nanoparticles for selective sensing of $\mathrm{Hg}^{2+}$ in food and cosmetics samples. RSC Adv. 2016, 6, 89916-89924.

(59) Huang, C.-C.; Yang, Z.; Lee, K.-H.; Chang, H.-T. Synthesis of highly fluorescent gold nanoparticles for sensing mercury(II). Angew. Chem. 2007, 119, 6948-6952.

(60) Klein, G.; Kaufmann, D.; Schurch, S.; Reymond, J.-L. A fluorescent metal sensor based on macrocyclic chelation. Chem. Commun. 2001, 561-562.

(61) Feng, J.; Chen, Y.; Han, Y.; Liu, J.; Ren, C.; Chen, X. Fluorescent carbon nanoparticles: A low-temperature trypsin-assisted preparation and $\mathrm{Fe}^{3+}$ sensing. Anal. Chim. Acta 2016, 926, 107-117.

(62) Liu, Y.; Gong, X.; Gao, Y.; Song, S.; Wu, X.; Shuang, S.; Dong C. Carbon-based dots co-doped with nitrogen and sulfur for $\mathrm{Cr}(\mathrm{vi})$ sensing and bioimaging. RSC Adv. 2016, 6, 28477-28483. 